\title{
ISOPHOT observations of dust discs around main sequence stars
}

\author{
H. J. Walker \\ Rutherford Appleton Laboratory, Chilton, Didcot OX11 OQX, UK
}

\begin{abstract}
The photometer (ISOPHOT) on the ESA Infrared Space Observatory (ISO) satellite was used to study the four Vega-like prototypes (Vega, $\beta$ Pic, $\alpha$ PsA, $\epsilon$ Eri) and a set of candidate stars believed to have similar dust/debris discs. Most of the candidate stars were main sequence stars, but probably younger than Vega and $\epsilon$ Eri since they showed residual emission in the cores of their stellar spectral lines. Low resolution spectra were combined with long wavelength multi-filter photometry (between $60 \mu \mathrm{m}$ and $200 \mu \mathrm{m}$ ) to give the spectral energy distributions for the dust/debris discs. Models suggested that the masses of the discs were between $10^{-7}$ and $10^{-4} \mathrm{M}_{\odot}(2-2000$ times the mass of the moon). These were more massive than the discs around the four prototypes, but less massive than the discs around young stars such as T Tau stars.
\end{abstract}

\section{Introduction}

The four prototype Vega-like stars (Vega, $\beta$ Pic, $\alpha$ PsA, $\epsilon$ Eri) have tenuous dust (or debris) discs around them. They are cool $(\mathrm{T}<100 \mathrm{~K})$, with large dust grains (larger than normal interstellar dust grains, hence the term debris discs), and they were first detected using IRAS (the Infrared Astronomical Satellite) by Aumann et al. (1984) and Gillett (1986). Only the disc around $\beta$ Pic has been detected optically (e.g. Smith \& Terrile 1984; Heap et al. 2000), and so follow-up has taken place in the infrared and sub-millimetre. The discs around Vega, $\beta$ Pic, $\alpha$ PsA were resolved at $850 \mu \mathrm{m}$ by Holland et al. (1998), and that around $\epsilon$ Eri by Greaves et al. (1998).

The photometer, ISOPHOT (see Lemke et al. 1996), on the ISO satellite (see Kessler et al. 1996) covered the wavelength range from $2.4 \mu \mathrm{m}$ to $240 \mu \mathrm{m}$, with several broad-band filters and low resolution spectroscopy from $2.5 \mu \mathrm{m}$ to $5 \mu \mathrm{m}$ and $5.8 \mu \mathrm{m}$ to $11.6 \mu \mathrm{m}$. In addition to the four prototypes, several mainsequence stars with excess infrared emission (from IRAS fluxes) from the Walker \& Wolstencroft (1988) list were investigated. The data were reduced using the ISOPHOT Interactive Analysis package PIA v7.3 (Gabriel et al. 1997) and for the low-resolution spectra the dynamic spectral response correction (Klaas et al. 1997) was used.

\section{Results}

The long wavelength photometry (at $60,80,100,120,150,170$ and $200 \mu \mathrm{m}$ ) was used to estimate a dust temperature and emissivity ('Emis' in Table 1) for 
the 12 objects observed in the programme, see Walker \& Heinrichsen (2000) for more information. Where possible the spectrum, as observed with ISOPHOT, is compared to other data. The dust mass is estimated using simple assumptions (see Heinrichsen et al. 1998).

Table 1. Characteristics of targets observed with ISOPHOT

\begin{tabular}{llllrrc}
\hline Star & Sp.Type & $\begin{array}{l}\text { ISOPHOT } \\
\text { spectrum }\end{array}$ & $\begin{array}{l}\text { Spectrum } \\
\text { type }\end{array}$ & $\begin{array}{r}\mathrm{T} \\
(\mathrm{K})\end{array}$ & $\begin{array}{r}\text { Emis } \\
\lambda^{n}\end{array}$ & $\begin{array}{c}\text { Dust mass } \\
\left(\mathrm{M}_{\odot}\right)\end{array}$ \\
\hline Vega & A0V & Photosphere & & 65 & -1.0 & $4.0 \times 10^{-9}$ \\
$\beta$ Pic & A5V & Cool thermal & Silicate $^{1}$ & 65 & -1.0 & $3.2 \times 10^{-8}$ \\
$\alpha$ PsA & A3V & Photosphere & & 55 & -1.0 & $8.4 \times 10^{-9}$ \\
$\epsilon$ Eri* & K2V & Photosphere & & 50 & -1.0 & $1.1 \times 10^{-9}$ \\
$49 \mathrm{Cet}$ & A3V & Cool thermal & & 60 & -1.0 & $5.5 \times 10^{-8}$ \\
HD98800* & K5Ve & Cool thermal & Silicate $^{2}$ & $45:$ & -2.0 & $6.7 \times 10^{-8}$ \\
HD139614 & A7Ve & Silicate & Silicate $^{3}$ & $45:$ & -1.5 & $4.5 \times 10^{-6}$ \\
HD135344 & F4Ve & Silicate & Silicate $^{4}$ & 50 & -1.1 & $8.1 \times 10^{-5}$ \\
HD144432 & A9/F0Ve & Silicate & Silicate $^{5}$ & 50 & -2.0 & $4.5 \times 10^{-6}$ \\
HD169142 & A5Ve & C-rich & C-rich $^{4}$ & 55 & -1.0 & $4.3 \times 10^{-5}$ \\
HD34700 & G0V & C-rich & C-rich $^{2}$ & 60 & -1.1 & $5.7 \times 10^{-7}$ \\
HD142666 & A8Ve & Si+C-rich & Si+C-rich $^{3}$ & 110 & 0.0 & $6.7 \times 10^{-6}$ \\
\hline \hline
\end{tabular}

Notes: Cochran (this symposium) reports the presence of a planet around $\epsilon$ Eri; HD98800 is a quadruple system (Koerner et al. 2000) with the dust disc around one pair of stars; ${ }^{1}$ Knacke et al. (1993); ${ }^{2}$ Sylvester \& Skinner (1996); ${ }^{3}$ Sylvester et al. (1997); ${ }^{4}$ Sylvester et al. (1995); ${ }^{5}$ Walker et al. (1997)

\section{Discussion}

Many of the spectral types for the stars came from Dunkin et al. (1997), and show that the programme stars are still young (compared to the four Vega-like prototypes), since they have residual emission in the line cores. A few of the stars are classed as Herbig $\mathrm{Ae} / \mathrm{Be}$ stars, although the disc masses determined here are possibly a little low for that evolutionary stage. Walker \& Wolstencroft (1988) reported non-detections of $\mathrm{CO}$ around the prototype and programme stars. Thi et al. (2001) have detected $\mathrm{H}_{2}$ emission (at $28 \mu \mathrm{m}$ ) from $\beta$ Pic, $49 \mathrm{Cet}$, and HD135344, with molecular hydrogen masses around 100 times the dust masses given here. The presence of molecular gas in the systems suggests that they can still be regarded as 'young', and as Lissauer (2001) remarks, the continuing presence of molecular gas means that there is more time to grow giant planets. Walker \& Heinrichsen (2000) noted that the disc around $\epsilon$ Eri was not resolved at $60 \mu \mathrm{m}$, but was resolved at $850 \mu \mathrm{m}$ (Greaves et al. 1998), showing that there may be cool outer regions to the discs where molecular gas would remain.

\section{References}

Aumann, H. H., et al. 1984, ApJ, 278, L23

Dunkin, S. K., Barlow, M. J., \& Ryan, S. G. 1997, MNRAS, 286, 604 
Gabriel, C., et al. 1997, in ASP Conf. Ser. Vol 125, ADASS VI, Conference on Astronomical Data Analysis Software and Systems (San Francisco: ASP), 108

Gillett, F. C. 1986, in Light on Dark Matter, ed. F. Israel (Dordrecht: Reidel), 61

Greaves, J. S., et al. 1998, ApJ, 506, L133

Heap, S. A., et al. 2000, ApJ, 539, 435

Heinrichsen, I., Walker, H. J., \& Klaas, U. 1998, MNRAS, 293, L78

Holland, W. S., et al. 1998, Nature, 392, 788

Kessler, M. F., et al. 1996, A\&A, 315, L27

Klaas, U., et al. 1997, in ESA-SP419, Proc. First ISO Workshop on Analytical Spectroscopy, eds. A. Heras, K. Leech, N. Trams, M. Perry (Noordwijk: ESA), 113

Knacke, R. F., Fajardo-Acosta, S.B., Telesco, C. M., Hackwell, J. A., Lynch, D. K., Russell, R. W. 1993, ApJ, 418, 440

Koerner, D. W., Jensen, E. L. N., Cruz, K. L., Guild, T. B., Gultekin, K. 2000, ApJ, $533, \mathrm{~L} 37$

Lemke, D., et al. 1996, A\&A, 315, L64

Lissauer, J. J. 2001, Nature, 409, 23

Sylvester, R. J., Barlow, M. J., \& Skinner, C. J. 1995, Ap\&SS, 224, 405

Sylvester, R. J., \& Skinner, C. J. 1996, MNRAS, 283, 457

Sylvester, R. J., Skinner, C. J., \& Barlow, M. J. 1997, MNRAS, 289, 831

Smith, B. A., \& Terrile, R. T. 1984, Science, 226, 1421

Thi, W.F., et al. 2001, Nature, 409, 60

Walker, H. J., Butner, H. M., Wooden, D., Witteborn, F. 1997, in The Role of Dust in the Formation of Stars, eds H. U. Käufl \& R. Siebenmorgen (Berlin: Springer), 223

Walker, H. J., \& Heinrichsen, I. 2000, Icarus, 143, 147

Walker, H. J., \& Wolstencroft, R. D. 1988, PASP, 100, 1509 